\title{
Unusual spontaneous improvement in Asian variant of intravascular large B-cell lymphoma
}

\author{
Takashi Ninomiya $^{1,2^{*}}$, Toru Nakamura ${ }^{2}$, Nobuharu Fujii ${ }^{1}$, Akio Hiraki ${ }^{1}$, Shigeki Umemura ${ }^{2}$, \\ Hiromichi Yamane ${ }^{2}$, Atsuko Shirakawa ${ }^{3}$, Haruhito Kamei ${ }^{2}$ \\ ${ }^{1}$ Department of Hematology, Oncology and Respiratory Medicine, Okayama University Hospital, Okayama, Japan \\ ${ }^{2}$ Department of Medicine, Sumitomo-Besshi Hospital, Niihama, Japan \\ ${ }^{3}$ Department of Pathology, Sumitomo-Besshi Hospital, Niihama, Japan \\ Email: ${ }^{\text {tninomiya5@gmail.com }}$
}

Received 23 January 2012; revised 15 February 2012; accepted 29 February 2012

\begin{abstract}
The clinical course of the Asian variant of intravascular large B-cell lymphoma (AIVL) is generally very aggressive. We describe a case of AIVL demonstrating an unusual clinical course, with spontaneous improvement. An 81-year-old man with high-grade fever and thrombocytopenia was admitted to our hospital. Although we could not confirm the origin of his symptoms, they disappeared completely without intervention within 2 weeks. Three months later, however, thrombocytopenia reappeared and progressed. Finally, he was readmitted due to a subdural hemorrhage with high fever and he finally died of rapidly progressive multiple organ failure. Autopsy findings revealed the presence of $B$-cell lymphoma cells in microscopic vessels of many organs as well as hemophagocytosis in the bone marrow. He was diagnosed with AIVL with an unusual indolent clinical course with spontaneous improvement.
\end{abstract}

Keywords: Lymphoma; Intravascular Large B-Cell Lymphoma; Spontaneous Improvement;

Hemophagocytosis

\section{INTRODUCTION}

Intravascular large B-cell lymphoma (IVLBCL) is a rare subtype of extranodal diffuse large B-cell lymphoma (DLBCL). Most Japanese cases of IVLBCL with hemophagocytic syndrome have been categorized as the "Asian variant" of IVLBCL (AIVL) [1]. The typical clinical course of AIVL is very progressive and spontaneous improvement is extremely rare. Here, we report an unusual indolent clinical course of AIVL demonstrating spontaneous improvement and mimicking low-grade lymphoma.

\footnotetext{
"Corresponding author.
}

\section{CASE REPORT}

An 81-year-old man was admitted to our hospital owing to high fever $>39^{\circ} \mathrm{C}$ in August 2006. Physical examination revealed hepatosplenomegaly with no other abnormal findings, such as lymph node swelling or skin rash. Laboratory data showed pancytopenia (white blood cell count (WBC), 2870/ $\mu \mathrm{L}$; hemoglobin (Hgb), $10.7 \mathrm{~g} / \mathrm{dL}$; platelet count (Plt), 21,000/ $\mu \mathrm{L}$ ), and elevation of serum lactate dehydrogenase (LDH, $779 \mathrm{IU} / \mathrm{L})$, C-reactive protein $(\mathrm{CRP}, 5.0 \mathrm{mg} / \mathrm{dL})$, and soluble interleukin-2 receptor (sIL-2 receptor, 10,800 U/mL). The results of other serological tests were within normal ranges, including liver enzyme and ferritin test. We performed chest and abdominal computed tomography (CT) (Figure 1) or magnetic resonance imaging of the brain; however, no evidence was found for any abnormality except hepatosplenomegaly. At that point, we suspected hemophagocytic syndrome, due to viral infection or malignant lymphoma, but neither malignant cells nor hemophagocytosis were detected after two individual aspirations and biopsies of the bone marrow, respectively. Additionally,

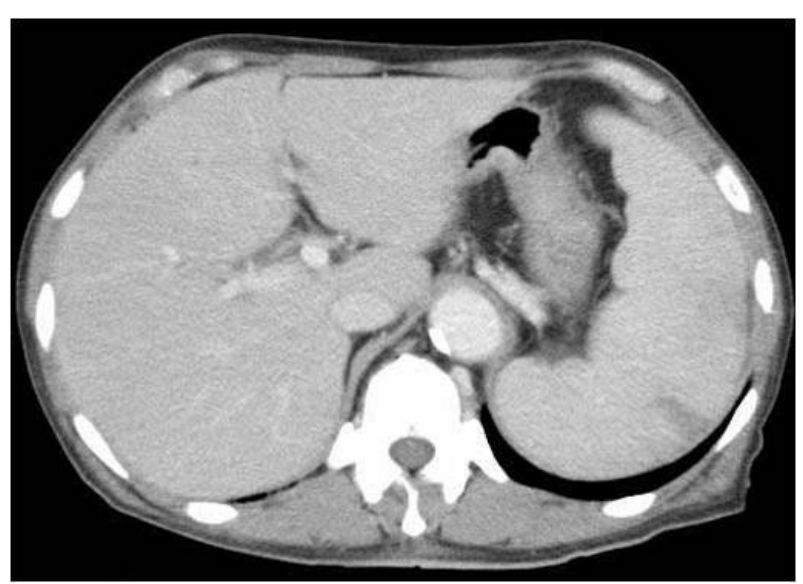

Figure 1. Abdominal computed tomography $(\mathrm{CT})$ at initial admission showing hepatomegaly and splenomegaly. 
no abnormality was observed following gallium scintigraphy. Fluorodeoxyglucose-positron emission tomography (FDG-PET) examination was not available in our hospital. The quantitative polymerase chain reaction (PCR) result of Epstein-Barr virus DNA in his serum was negative $\left(<2 \times 10^{2}\right.$ copies $\left./ \mathrm{mL}\right)$. We thought that IVLBCL was still a possibility and considered performing a liver biopsy. However, his fever had suddenly normalized and his LDH and platelet levels had become normal without therapy on day 14 after admission. Thus, we excluded the possibility of IVLBCL and did not perform a liver biopsy; we suspected that he had suffered from a viral infection. Finally, he was discharged in October 2006 and was followed as an outpatient.

His fever with thrombocytopenia and elevation of LDH levels gradually reappeared in December 2006. In February 2007, he was readmitted to our hospital owing to a consciousness disorder. On admission, he was diagnosed with acute subdural hematoma by head CT (Figure 2). His hematoma was treated conservatively and progression was not observed; unfortunately, however, his consciousness disorder did not improve. Eight days after readmission, a high fever, $>39^{\circ} \mathrm{C}$, appeared. He had pleural effusions and ascites as well as hepatosplenomegaly, without lymphadenopathy (Figure 3). A blood examination revealed that his LDH and sIL-2 receptor levels were substantially elevated (LDH, 1,186 IU/L; sIL-2 receptor, 35,500 U/mL) and hyperuricemia was also present. His hepatic and renal injuries progressed rapidly, and he died of multiple organ failure in March 2007 (Figure 4).

Autopsy findings showed that lymphoma cells were present in microscopic vessels and sinusoids of his lung, myocardium, thyroid glands, adrenal glands, liver, kidneys, prostatic glands, pancreas, spleen, and gastrointes

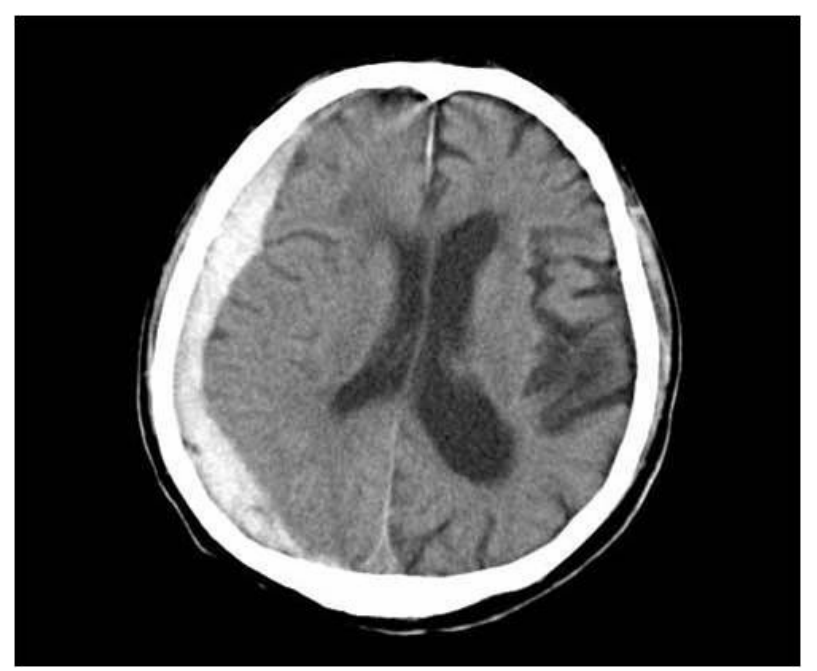

Figure 2. Head CT at readmission showing right subdural bleeding.
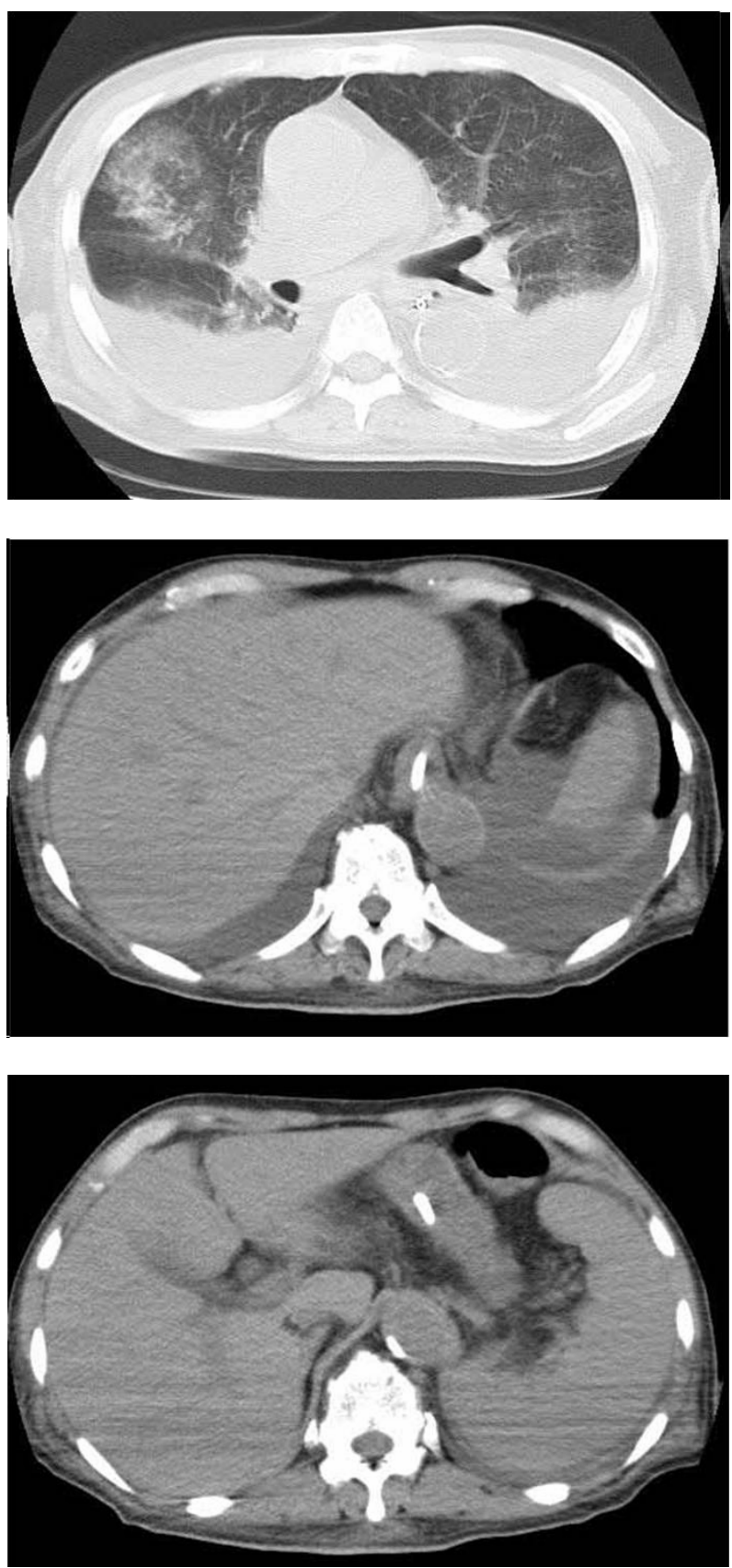

Figure 3. Chest and abdominal $\mathrm{CT}$ at 8 days after readmission showing pleural effusion, ascites, and hepatosplenomegaly.

tinal tract (Figure 5). Additionally, hemophagocytosis was evident in the bone marrow. Immunohistochemical studies indicated that the phenotypes of the lymphoma cells were positive for bc12, CD20, and CD79a, but negative for CD10, CD56, and CD5. Finally, he was diagnosed with AIVL.

\section{DISCUSSION}

IVLBCL is a rare subtype of extranodal DLBCL characterized by massive proliferation of large tumor cells 


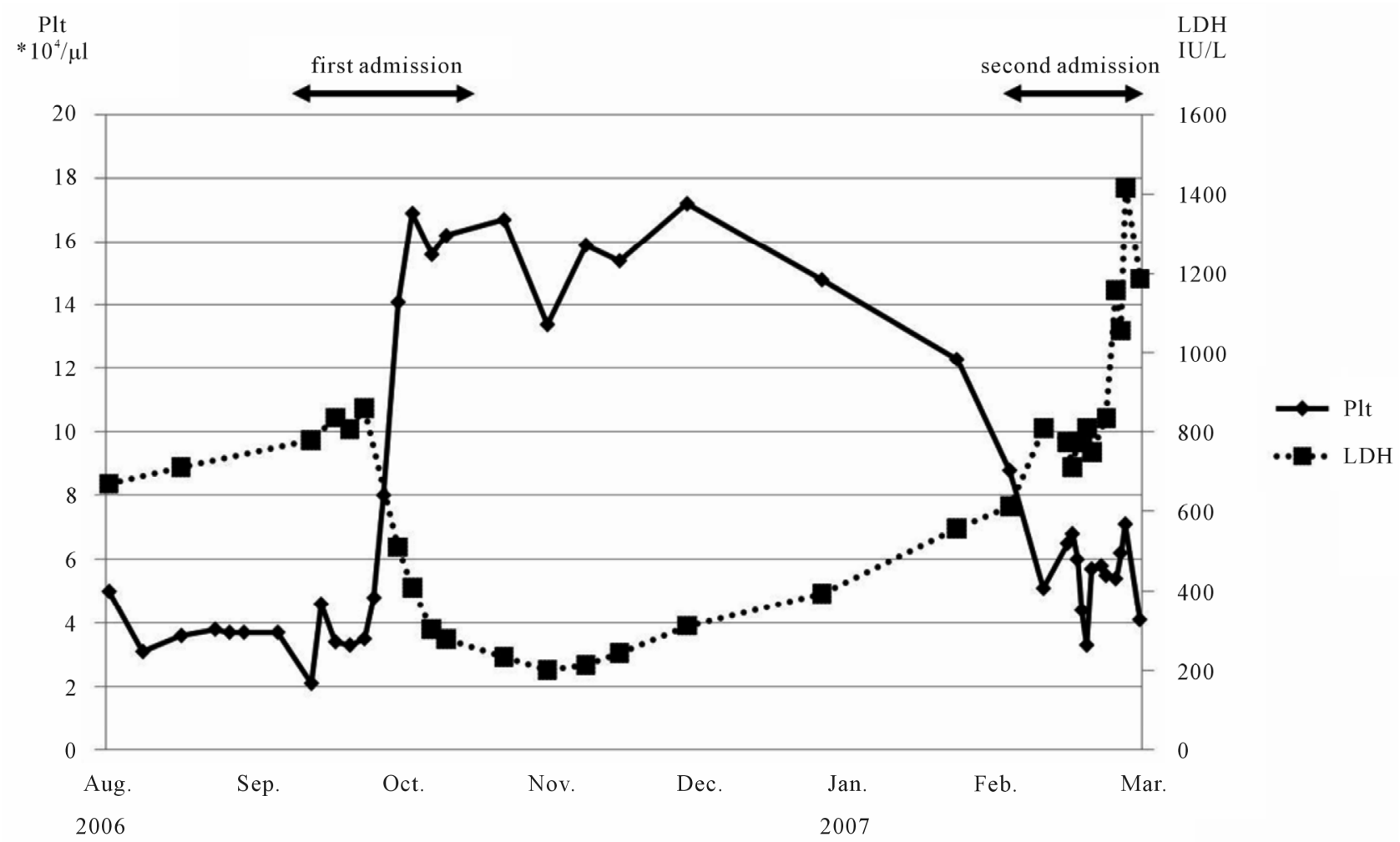

Figure 4. Clinical course and fluctuation of platelet and serum lactate dehydrogenase levels.

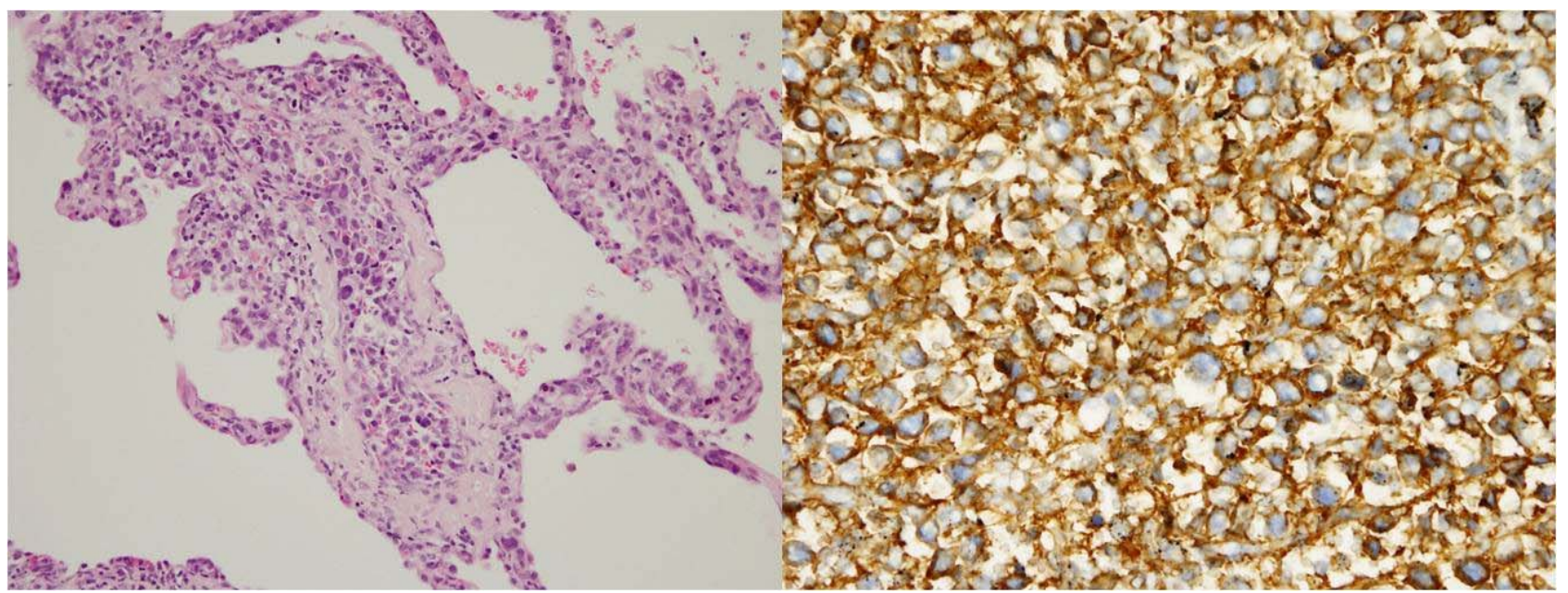

Figure 5. Pathological lung specimen: malignant lymphoma cells fill a small vessel (hematoxylin and eosin staining; magnification, $\times 400$ ). Malignant lymphoma cells (immunochemical staining for CD20).

within the lumina of small-to-medium-sized vessels [2]. Highly variable symptoms, due to tumor occlusion of microvessels in various organs, have been reported. $\mathrm{Mu}-$ rase et al. reported that most Japanese cases were associated with hemophagocytic syndrome and these cases were recognized as AIVL. They proposed diagnostic criteria for AIVL $[1,3,4]$ and reported a higher incidence of hepatosplenomegaly, bone marrow invasion, and thrombocytopenia in AIVL. Conversely, neurological and dermatological signs that were specific to IVLBCL in Western countries were not common in a study of 96 Japanese IVLBCL patients [1].

In our case, B-cell lymphoma cells were evident only in microscopic vessels and sinusoids and hemophagocytosis was observed in the bone marrow. Although hepatosplenomegaly was observed, lymphadenopathy was not apparent throughout the clinical course. Because cytopenia of erythrocytes and platelets was evident, he was 
diagnosed with AIVL on the basis of Murase's criteria for AIVL [2-4]. Although we could not obtain histopathological confirmation of malignant lymphoma or hemophagocytosis on the first admission, his symptoms were suspected to be caused by AIVL.

The clinical course of AIVL is typically very aggressive. However, a few reports have demonstrated an indolent type of IVLBCL. Ishiko et al. reported that a 59year-old woman with ataxia and gait disturbance after a 3-year history of serum LDH elevation was finally diagnosed with IVLBCL by brain biopsy [5]. They suggested that there are certain IVLBCL patients with brain involvement who present with relatively slow progression, similar to a low-grade lymphoma [5]. Nakao et al. reported that a 62-year-old woman with slowly developing paraplegia and a 7-month history of recto-urinary dysfunction was diagnosed with IVLBCL by autopsy [6]. In that case, lymphoma cells were only identified in the bone marrow and blood vessels of the cauda equina. Additionally, Sekine et al. reported that a 71-year-old man with respiratory failure after developing intermittent fever and a high serum LDH for 7 months was diagnosed with IVLBCL by lung biopsy [7]. In that case, lymphoma cells were identified in the cauda equina and lung. From these different lines of evidence, we suggest that IVLBCL may have a slow progressive clinical course when lymphoma cells are present in limited organs. Although we could not show the limited lymphoma cell involvement in a specific organ, our case might be one of indolent AIVL similar to these previous reported cases.

In two of three previous cases, a diagnosis of IVLBCL was established in living patients who were treated using CHOP (cyclophosphamide, doxorubicin, vincristine, prednisone) with rituximab and showed complete remission. In the other case and in our case, the diagnosis was established at autopsy. IVLBCL is difficult to diagnose because patients generally show little or no tumor mass formation and more than half of patients are diagnosed postmortem. Recently, some reports have suggested that a random skin biopsy may be efficacious for the early diagnosis of IVLBCL. Asada et al. reported that early diagnosis of IVLBCL was possible by random skin biopsy and the early institution of rituximab-based chemotherapy induced a favorable response [8]. In our case, a liver biopsy or random skin biopsy should have been performed even after the high fever and thrombocytopenia disappeared.

In conclusion, we present a case of AIVL with an unusual clinical course and spontaneous improvement. We suggest that an indolent clinical course or spontaneous improvement does not exclude AIVL, although most AIVL cases are aggressive and progressive. Importantly, AIVL should be treated immediately with intensive examination for diagnosis, even though the clinical course is apparently indolent.

\section{REFERENCES}

[1] Murase, T., Yamaguchi, M., Suzuki, R., Okamoto, M., Sato, Y., Tamaru, J., Kojima, M., Miura, I., Mori, N., Yoshino, T. and Nakamura, S. (2007) Intravascular large B-cell lymphoma (ivlbcl): A clinicopathologic study of 96 cases with special reference to the immunophenotypic heterogeneity of cd5. Blood, 109, 478-485.

doi:10.1182/blood-2006-01-021253

[2] Murase, T. and Nakamura, S. (1999) An Asian variant of intravascular lymphomatosis: An updated review of malignant histiocytosis-like B-cell lymphoma. Leukemia and Lymphoma, 33, 459-473.

[3] Murase, T., Nakamura, S., Kawauchi, K., Matsuzaki, H., Sakai, C., Inaba, T., Nasu, K., Tashiro, K., Suchi, T. and Saito, H. (2000) An Asian variant of intravascular large B-cell lymphoma: Clinical, pathological and cytogenetic approaches to diffuse large B-cell lymphoma associated with haemophagocytic syndrome. British Journal of Haematology, 111, 826-834.

doi:10.1046/j.1365-2141.2000.02426.x

[4] Murase, T., Nakamura, S., Tashiro, K., Suchi, T., Hiraga, J., Hayasaki, N., Kimura, M., Murakami, M., Mizoguchi, Y., Suzuki, T. and Saito, H. (1997) Malignant histiocytosis-like B-cell lymphoma, a distinct pathologic variant of intravascular lymphomatosis: A report of five cases and review of the literature. British Journal of Haematology, 99, 656-664. doi:10.1046/j.1365-2141.1997.4623265.x

[5] Ishiko, J., Mizuki, M., Yasumi, M., Ujiie, H., Nakamichi, I., Aozasa, K. and Kanakura, Y. (2007) An indolent subtype of "Intravascular lymphoma": A case with a 3-year history of ldh elevation. Leukemia and Lymphoma, 48, 1872-1874. doi:10.1080/10428190701493936

[6] Nakao, N., Yoshida, M., Iwata, M., Hashizume, Y. and Sahashi, K. (2008) A case of intravascular malignant lymphomatosis presenting as slowly progressive paraplegia. Brain Nerve, 60, 181-185.

[7] Sekine, A., Hagiwara, E., Okudera, K., Baba, T. and Ogura, T. (2009) A case of slowly progressive intravascular lymphoma with respiratory failure caused by diffuse pulmonary vasoconstriction. Nihon Kokyuki Gakkai Zasshi, 47, 924-929.

[8] Asada, N., Odawara, J., Kimura, S., Aoki, T., Yamakura, M., Takeuchi, M., Seki, R., Tanaka, A. and Matsue, K. (2007) Use of random skin biopsy for diagnosis of intravascular large B-cell lymphoma. Mayo Clinic Proceedings, 82, 1525-1527. doi:10.4065/82.12.1525 